\title{
KARAGABI KMMODEL: MODELO DE REFERENCIA PARA LA INTRODUCCIÓN DE INICIATIVAS DE GESTIÓN DEL CONOCIMIENTO EN ORGANIZACIONES BASADAS EN CONOCIMIENTO
}

\section{KARAGABI KMMODEL: REFERENCE MODEL FOR THE INTRODUCTION OF KNOWLEDGE MANAGEMENT INITIATIVES IN KNOWLEDGE BASED ORGANIZATIONS}

\author{
Alberto de J. González ${ }^{1} \quad$ Caroll Z. Joaquí $^{1} \quad$ Cesar A. Collazos $^{2}$ \\ Recibido 16 de junio de 2008, aceptado 15 de abril de 2009 \\ Received: June 16, 2008 Accepted: April 15, 2009
}

\begin{abstract}
RESUMEN
La inserción de las organizaciones en la economía del conocimiento demanda de ellas una reestructuración que les permita integrar y explotar el conocimiento en su operación convencional, y convertirlo en un verdadero activo estratégico. El desarrollo de nuevas competencias, estrategias, estructuras, procesos orientados a conocimiento y su gestión es fundamental para soportar las nuevas tendencias del mercado. Para responder a estas necesidades se deben desarrollar nuevas propuestas, metodologías, métodos, estándares para el diseño, evaluación y mejora de organizaciones orientadas a conocimiento. Este artículo presenta el modelo Karagabi KMModel cuyo objetivo es ser una guía de referencia para la construcción de organizaciones orientadas a conocimiento. Karagabi KMModel está integrado por una metodología de intervención que permite orientar el proceso de diseño de este tipo de organizaciones, una librería de modelos de conocimiento, que permiten representar conocimiento sobre distintos aspectos relevantes de la organización y una base de conocimiento de experiencias que permite la gestión de experiencias basada en casos de aplicación del mismo modelo. Se presentan también algunos ejemplos que han contribuido a conformar la base de conocimiento de experiencias, facilitando su aprovechamiento para trabajos futuros y el enriquecimiento del propio modelo.
\end{abstract}

Palabras clave: Gestión del conocimiento, ingeniería del conocimiento, sistemas basados en conocimiento, modelado organizacional, gestión por procesos del negocio, ontologías.

\begin{abstract}
The inclusion of the organizations into the knowledge economy requires reorganization that allows them to integrate the knowledge in their conventional operation in order to convert it in a true strategic active. Developing new knowledge and knowledge management oriented to competences, strategies, structures, and processes is essential to support the new trends. To meet these needs, it is necessary to define new proposals, methodologies, methods and standards for the design, evaluation and improvement of knowledge-oriented organizations. This paper presents Karagabi KMModel whose main goal is to serve as a reference guide for the construction of knowledge oriented organizations. Karagabi KMModel is composed of an intervention methodology that guides the design processes of these kind of organizations; a library of knowledge models, which represent knowledge about different aspects of the organization; and an experience knowledge base that allows experience management, based on application cases of the same model. Some examples of application are also presented, which have served to shape the experience knowledge base, facilitating its use for future work and improving the model itself.
\end{abstract}

Keywords: Knowledge management, Knowledge Engineering, Knowledge Based Systems, Organizational modelling, Business Process Management, Ontologies.

\footnotetext{
1 Empresa TOTEMS Ltda, Cauca, Colombia. E-mail: agonzalez@totems-software.com; cjoaqui@totems-software.com
}

2 Universidad del Cauca. Grupo IDIS. Cauca, Colombia. E-mail: ccollazo@unicauca.edu.co 


\section{INTRODUCCIÓN}

La gestión del conocimiento ha sido identificada como uno de los factores clave para el desarrollo, sostenimiento y competitividad de las organizaciones [1-3]. Su propósito principal es lograr que el conocimiento sea reconocido y aprovechado como cualquier otro activo de negocio, como algo que se utiliza, mantiene y distribuye en beneficio de la organización.

La inserción de las organizaciones en la economía del conocimiento demanda de ellas una reestructuración que les permita integrar y explotar el conocimiento en su operación convencional. Las organizaciones orientadas a conocimiento requieren nuevas estructuras, cambios culturales profundos, nuevas plataformas tecnológicas y modelos para la estructuración del conocimiento y de los procesos que permiten gestionarlo. Sin embargo, los modelos, metodologías, estándares existentes para el diseño, despliegue, evaluación y mejora de estrategias, estructuras y procesos organizacionales se quedan cortos para soportar las nuevas tendencias. La dimensión del conocimiento, y aun más el enfoque hacia la gestión del conocimiento no está presente, y no se reconocen lineamientos claros sobre la forma de hacer un uso más eficiente del conocimiento, ni como reconocerlo e integrarlo como un elemento clave que interviene en los procesos convencionales.

Por otra parte, los equipos responsables del diseño y despliegue de estrategias, procesos y estructuras organizacionales de la gestión del conocimiento deben enfrentarse a organizaciones heterogéneas, disímiles, en ambientes que cambian con mucha rapidez, debido a las condiciones del mercado, los clientes, sus necesidades, y la competencia; características que hacen que no exista una forma universal de gestionar conocimiento. Cada organización debe diseñar y aplicar su propia propuesta y disponer de instrumentos que permitan hacerle seguimiento, evaluarla y evolucionarla.

Es fundamental entonces bajo esta perspectiva la definición clara de métodos, metodologías, herramientas para apoyar a los equipos responsables de la gestión del conocimiento, en el diseño de estrategias, procesos, estructuras orientadas al conocimiento y su gestión, que permitan convertirlo en un activo estratégico al integrarlo de manera efectiva en la operación diaria de la organización.

Este artículo presenta el modelo Karagabi KMModel, cuyo objetivo es ser una guía de referencia para la construcción de organizaciones orientadas a conocimiento. Karagabi KMModel está integrado por una metodología de intervención que permite guiar el proceso de diseño de la organización orientada a conocimiento; una librería de modelos de conocimiento que permiten representar conocimiento sobre distintos aspectos relevantes de la organización; y una base de conocimiento de experiencias que permite la gestión de experiencias de aplicación del modelo. Karagabi KMModel integra las teorías y prácticas de gestión y modelado organizacional [4-6], la ingeniería del conocimiento [7], ingeniería de ontologías [8], los sistemas basados en el conocimiento, la gestión del conocimiento y la gestión por procesos del negocio [9] (BPM). Su aplicación en distintos proyectos ha permitido evaluar los componentes del modelo y plantear perspectivas de trabajo futuro.

El artículo se ha estructurado de la siguiente forma. La primera sección presenta una descripción general del modelo Karagabi KMModel. Las siguientes secciones presentan los componentes del modelo, su metodología de intervención, la librería de modelos de conocimiento y la base de conocimiento de experiencias. Finalmente se presentan algunos casos de aplicación del modelo y las conclusiones y perspectivas de trabajo futuro.

\section{DESCRIPCIÓN GENERAL DE KARAGABI KMMODEL}

\section{Referentes para la definición de Karagabi KMModel}

La definición de modelos, métodos, técnicas, y herramientas de gestión del conocimiento y su integración a los modelos y metodologías convencionales para el diseño y despliegue de organizaciones orientadas a conocimiento, es en la actualidad una temática que ha generado gran interés entre los grupos de investigación y empresas en el área de ingeniería del conocimiento, gestión organizacional y gestión del conocimiento a nivel mundial. Proyectos y modelos de gestión del conocimiento como los desarrollados en CommonKads [7], METIS [10], OntoKnowledge [11], DECOR [12], Know-Net [13], VTT Electronics [14] han hecho aportes importantes en esta temática y constituyen los principales referentes de Karagabi KMModel. Estos proyectos persiguen la construcción de un marco conceptual y práctico para el diseño, despliegue y seguimiento de iniciativas de gestión del conocimiento organizacional, basados en las teorías y prácticas de modelado organizacional, la ingeniería del conocimiento, la gestión por procesos del negocio y la gestión organizacional. Otras propuestas relacionadas con la valoración de conocimiento [15], los modelos de madurez en gestión de conocimiento [16-18], la medición del capital intelectual [19-20], la gestión por procesos del negocio, la gestión del talento humano 
por competencias [21-22], la auditoría de conocimiento [23-24], la construcción de mapas de conocimiento [2526], y la gestión de experiencias [27] han constituido un punto de partida para direccionar elementos particulares de Karagabi KMModel.

\section{Características fundamentales de Karagabi KMModel}

Karagabi KMModel provee un marco de trabajo integral, para el diseño, despliegue, evaluación y mejora de modelos organizacionales orientados a la gestión del conocimiento. Tres componentes fundamentales forman la base del modelo propuesto (ver figura 1), la metodología de intervención, la librería de modelos de conocimiento, y la base de conocimiento de experiencias. La metodología de intervención define un conjunto de procesos y actividades de referencia para orientar el diagnóstico, diseño, despliegue, seguimiento, evaluación y mejora de organizaciones orientadas a conocimiento. La librería de modelos de conocimiento está integrada por modelos ontológicos que facilitan la identificación, estructuración y almacenamiento de conocimiento sobre diferentes aspectos relevantes de la organización en las distintas fases de la metodología de intervención. La librería de modelos de conocimiento está dividida en siete grupos o paquetes; los modelos núcleo, que incluyen el modelo de estructura, el modelo motivacional, el modelo de procesos del negocio, orientados a conocimiento y el modelo del talento humano orientado a competencias; los modelos de experticia; los modelos de diagnóstico y análisis; los modelos de valoración; los modelos de soporte; los modelos de conocimiento de unidades organizacionales específicas, y los modelos de conocimiento de dominios específicos. La base de conocimiento de experiencias permite gestionar el conocimiento sobre Karagabi KMModel de forma integral, estructurando y almacenando experiencia en la forma de casos de aplicación, conocimiento de expertos y conocimiento relativo al cambio del propio modelo.

Karagabi KMModel está caracterizado por:

- Karagabi KMModel está basado en las teorías y prácticas de modelado y gestión organizacional, permitiendo integrar las estrategias, procesos y estructuras de la gestión del conocimiento a los modelos de gestión convencionales. Es fundamental la integración a Karagabi KMModel del modelo de gestión del talento humano por competencias como una de las principales estrategias de gestión de organizaciones orientadas a conocimiento.

- Karagabi KMModel integra instrumentos desde las distintas propuestas existentes para la gestión de organizaciones orientadas a conocimiento.

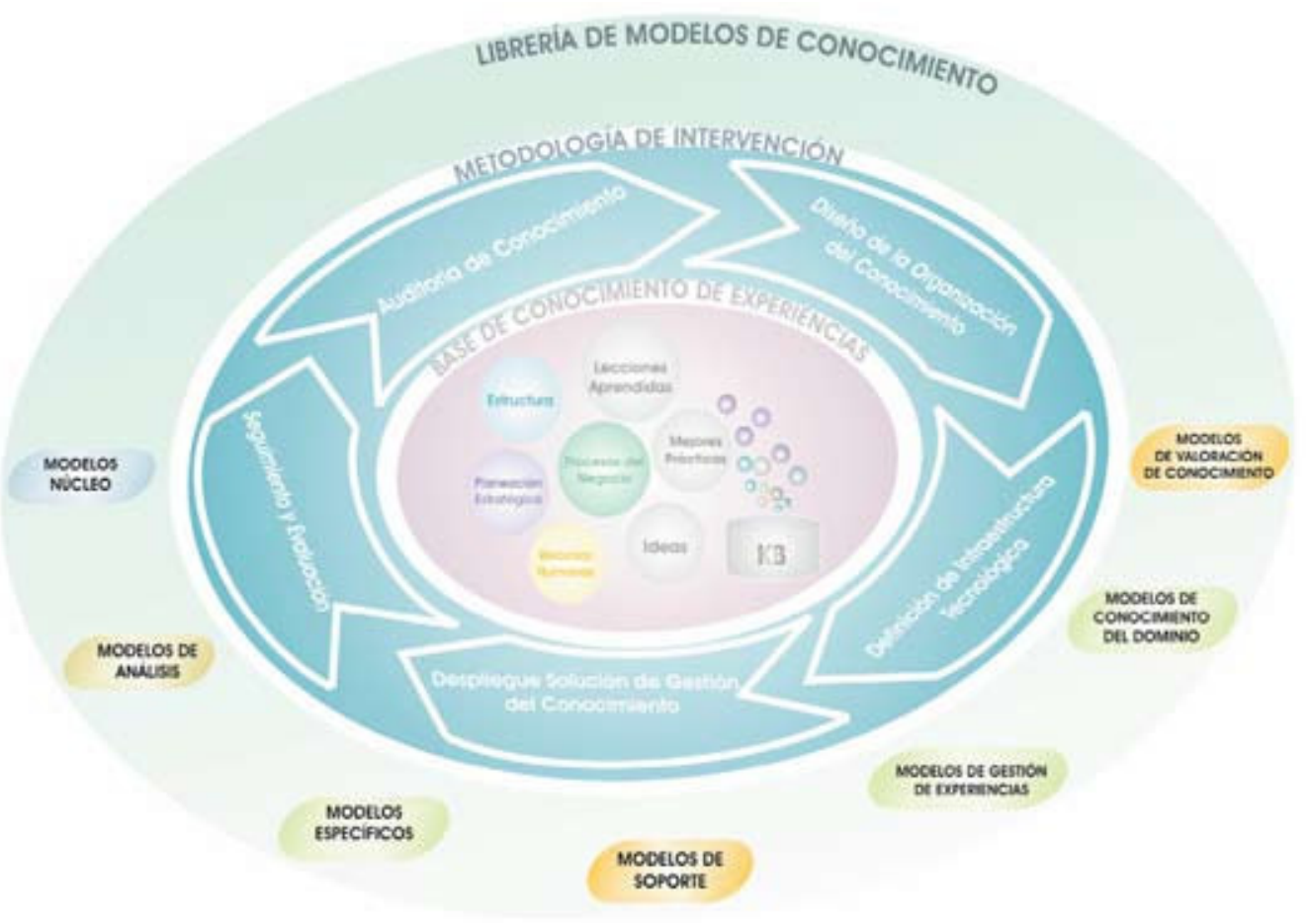

Figura 1. Componentes de Karagabi KMModel. 
Los modelos de madurez, de métricas e indicadores de gestión del conocimiento, de valoración de capital intelectual, gestión del cambio, gestión de experiencias, gestión por procesos del negocio se convierten en un insumo fundamental para el diseño y construcción de tales organizaciones.

- Karagabi KMModel está basado en modelos de conocimiento que permiten describir diferentes aspectos que conforman la organización orientada al conocimiento, como su estructura, sus procesos, personas, tecnología, y motivación. Los modelos de conocimiento permiten que todos los actores involucrados (desde administradores, hasta ingenieros de software) tengan un entendimiento común y compartido de su organización. Estos modelos son claves para hablar el mismo lenguaje en las diferentes fases de la metodología de intervención, y sirven para representar los aspectos a diagnosticar, diseñar y evaluar.

- Los modelos de conocimiento en Karagabi KMModel están basados en estándares internacionales, definidos por organismos de estandarización como $\mathrm{ISO}^{3}, \mathrm{OMG}^{4}$, $\mathrm{WFMC}^{5}, \mathrm{OASIS}^{6}, \mathrm{IDEF}^{7}$, lo que facilita su adopción, adaptación e integración a organizaciones, sistemas y servicios.

- Karagabi KMModel integra las teorías y prácticas de la ingeniería del conocimiento y la ingeniería de ontologías para la creación y gestión de su librería de modelos de conocimiento. Los modelos existentes pueden ser especializados y extendidos y nuevos modelos pueden ser desarrollados para soportar distintas perspectivas de gestión del conocimiento y/o estructurar conocimiento de dominios específicos.

- Una de las principales contribuciones de Karagabi KMModel corresponde a su base de conocimiento de experiencias, que permite la construcción colaborativa de conocimiento basado en casos y la gestión del cambio de su propia metodología de intervención. La experiencia almacenada permite hacer cada vez más robusto el modelo y por lo tanto reducir tiempos y costos en la introducción de iniciativas de gestión del conocimiento.

\footnotetext{
3 International Organization for Standardization, http://www.iso.org

4 Object Management Group, http://www.omg.org

5 Work Flow Management Coalition. http://www.wfmc.org

6 Organization for the Advancement of Structured Information Standards. http:// www.oasis-open.org

7 Integrated Definition Methods. http://www.idef.com
}

- Karagabi KMModel es un modelo adaptable. El conjunto de procesos, instrumentos y modelos de conocimiento definidos, se convierten en un referente para los consultores los cuales pueden replantear fases y actividades de la metodología de intervención, adicionar modelos de conocimiento a la librería y nuevo conocimiento a la base de conocimiento de experiencias.

\section{DEFINICIÓN DE LA METODOLOGÍA DE INTERVENCIÓN}

Karagabi KMModel orienta un proceso estructurado para el diseño de modelos de gestión de conocimiento específicos para las organizaciones, adaptados a sus necesidades, a sus problemas y oportunidades y a sus restricciones a través de su metodología de intervención, la cual define un conjunto de fases y actividades base que incluyen la auditoría de conocimiento, el diseño de la organización orientada a conocimiento, la definición de la infraestructura tecnológica de soporte, el despliegue del sistema de gestión del conocimiento y su seguimiento y evaluación (ver figura 2).

\section{Auditoría de conocimiento}

Consiste en el análisis sistemático de los procesos, información y los activos de conocimiento de una organización y sus atributos claves, como los propietarios, su uso y los flujos, los cuales son mapeados en función de las necesidades de conocimiento de la organización. El proceso de auditoría permite la identificación de problemas y oportunidades de conocimiento y se convierte en la base para la definición de planes, metas, objetivos, estrategias, tácticas, orientadas a conocimiento, y para la definición de estructuras, procesos, roles, y competencias para soportar el cumplimiento de tales planes. Define una serie de subprocesos, que incluyen la definición del alcance, la construcción del mapa de conocimiento de la organización, el análisis de la gestión del conocimiento y el análisis de la cultura de gestión del conocimiento y su posible impacto sobre el despliegue de las estrategias y cursos de acción futuros (ver figura 3). Los modelos de análisis de la librería de modelos de conocimiento son fundamentales para adelantar este proceso. Los modelos núcleo y de soporte también son integrados como instrumentos que pueden ser empleados por el equipo que realiza la auditoría para la captura de conocimiento relevante. 


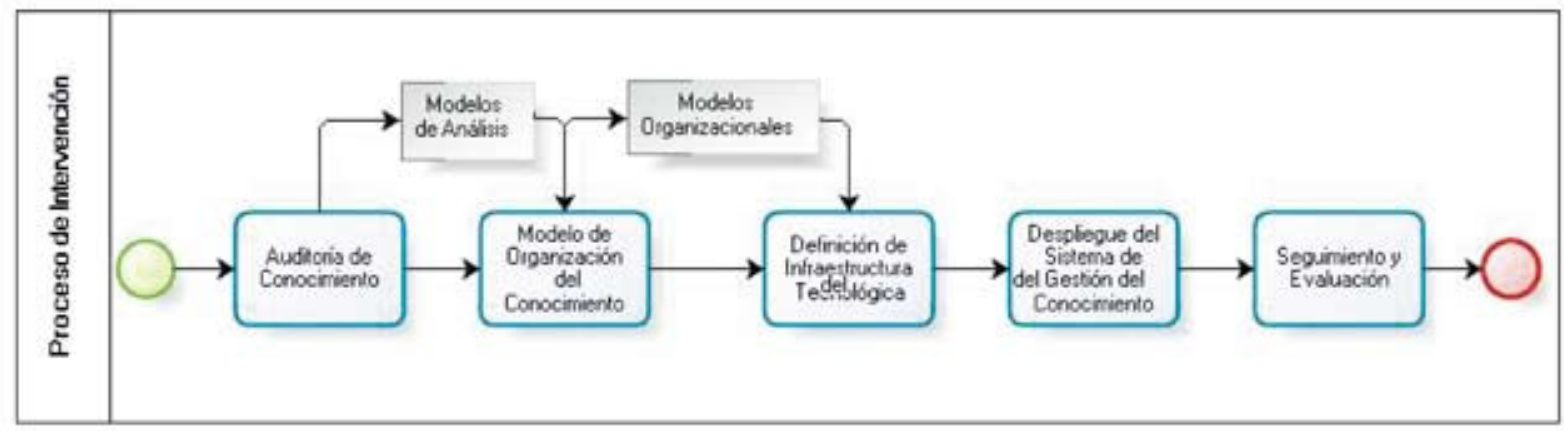

Figura 2. Proceso de intervención.

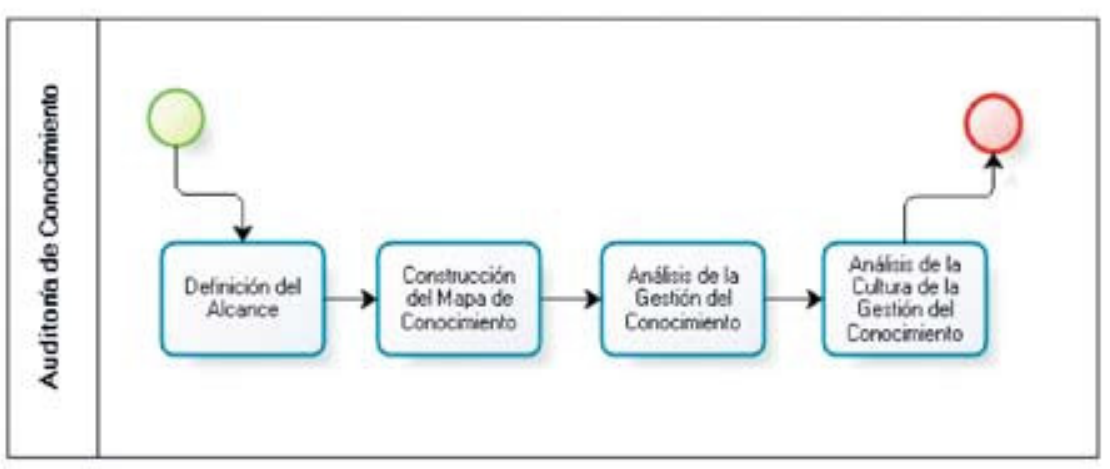

Figura 3. Proceso de auditoría.

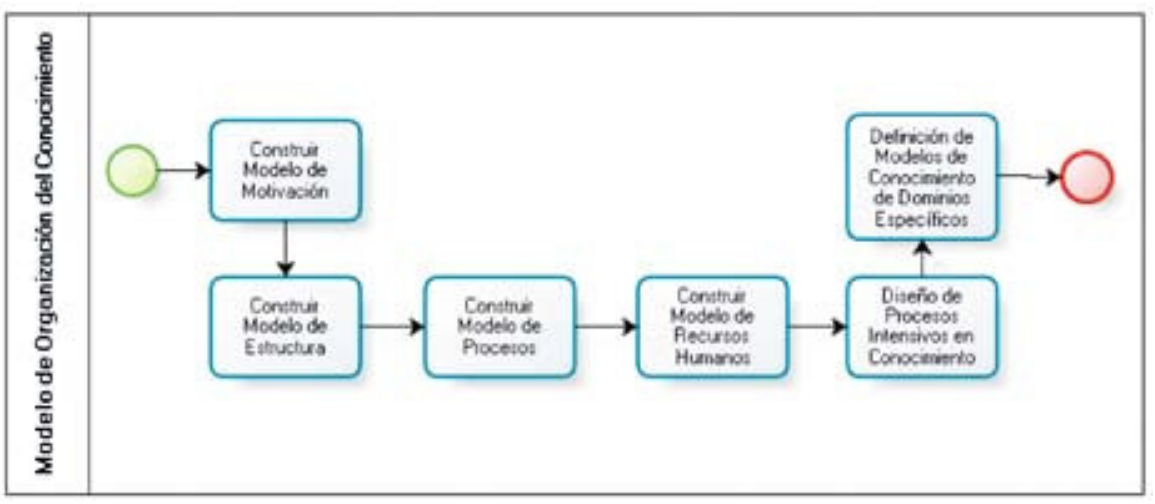

Figura 4. Proceso de modelado de organización del conocimiento.

\section{Diseño de la organización del conocimiento}

El proceso de diseño de la organización del conocimiento permite definir las estrategias, estructuras y procesos de gestión del conocimiento, así como el mapa de competencias, y los modelos de conocimiento específico del negocio de la organización. La principal entrada a este proceso corresponde al conocimiento generado al aplicar los modelos de análisis durante el proceso de auditoría de conocimiento. Los modelos núcleo de la librería de modelos de conocimiento son el principal instrumento para orientar la construcción de la organización orientada a conocimiento. La base de conocimiento de experiencias de Karagabi KMModel se constituye en un soporte fundamental, al proveer conocimiento de expertos, así como de casos anteriores en la ejecución del proceso con instancias particulares de estrategias, estructuras, procesos, y la asociación de lecciones aprendidas, problemas y soluciones que permitirán reducir los tiempos y costos relacionados. El diseño de la organización orientada al conocimiento se realiza a través de una serie de subprocesos que incluyen la construcción del modelo de motivación, la construcción del modelo de estructura, la construcción del modelo de procesos, la construcción del modelo de 
recursos humanos, el diseño de procesos intensivos en conocimiento y la definición de modelos de conocimiento de dominios específicos (ver figura 4).

\section{Definición de la infraestructura tecnológica de soporte a la gestión del conocimiento}

En esta fase se especifican las tecnologías que permiten soportar los procesos de gestión del conocimiento de la organización, para almacenamiento, acceso y gestión. La entrada principal de este proceso corresponde al conocimiento capturado a partir de los modelos de motivación y de procesos durante la etapa previa. Entre las actividades que deben ser ejecutadas durante este proceso se incluyen el análisis sobre cómo la tecnología puede ser empleada para el logro de las metas y el cumplimiento de los objetivos, sobre cómo la tecnología puede ser empleada para automatizar los procesos de gestión del conocimiento y la definición de las tecnologías y herramientas que pueden ser usadas [14].

\section{Despliegue del sistema de gestión del conocimiento}

El sistema de gestión del conocimiento debe ser desplegado a partir de la realización de pruebas piloto que permitan tener un espacio de validación, seguimiento, y evaluación controlado sin incurrir en grandes inversiones que pueden no ser convenientes. Como resultado de este ejercicio se generan recomendaciones sobre posibles mejoras en las estrategias, procesos, estructuras para su posterior despliegue al resto de la organización. Como entradas a este proceso se consideran el conocimiento capturado a partir de los modelos de análisis, los modelos de la organización orientada a conocimiento, y los modelos de infraestructura tecnológica.

\section{Seguimiento y evaluación}

Los procesos de seguimiento y evaluación soportan el mejoramiento continuo de la gestión del conocimiento organizacional. A partir del modelo de indicadores para la gestión del conocimiento se observan los impactos del despliegue de las estrategias y de los procesos que las soportan, en los indicadores de gestión de la organización. El resultado de este proceso es un insumo fundamental para la toma de decisiones sobre el despliegue de nuevas iniciativas de gestión del conocimiento o el mejoramiento de las existentes.

\section{LIBRERÍA DE MODELOS DE CONOCIMIENTO}

La librería de modelos de conocimiento está integrada por siete grupos o paquetes de modelos orientados según las perspectivas de conocimiento organizacional que direccionan y su aplicación en los procesos definidos dentro de la metodología de intervención. Los modelos de conocimiento son diseñados y construidos basados en las teorías y prácticas de la ingeniería del conocimiento y la ingeniería de ontologías lo que permite gestionar la evolución de la librería a través de la construcción de nuevos modelos, o la extensión o especialización de los ya existentes para adecuarlos a características particulares de las organizaciones objetivo. Los modelos son definidos en tres niveles distintos que permiten su apropiación a nivel conceptual, formal o como ontologías implementadas para ser integradas en herramientas de soporte a la gestión del conocimiento. A continuación se describen los siete paquetes de modelos de conocimiento:

\section{Modelos núcleo}

Los modelos núcleo estructuran conocimiento sobre los aspectos medulares del conocimiento organizacional incluyendo la motivación, los procesos, la estructura, el talento humano y la infraestructura tecnológica. Cinco modelos han sido definidos: (1) El modelo de motivación (BMKM) permite capturar, comunicar y gestionar la visión de la organización, sus metas y objetivos, los factores de influencia, así como sus estrategias, tácticas y políticas [28]. El modelo de motivación de la organización orientada a conocimiento es la base para la definición de sus procesos del negocio y su estructura; (2) El modelo de procesos del negocio (BPKM) permite expresar conocimiento sobre la operación de una organización particular en la forma de procesos, actividades, eventos del mundo real, objetos que participan en el proceso, objetos de soporte y las relaciones de precedencia y causalidad entre procesos y eventos en el ambiente; (3) El modelo de estructura (OSKM) permite estructurar el conocimiento de la organización sobre sus Unidades Organizacionales, Posiciones, Asignaciones, Entidades Legales; (4) El modelo del Talento Humano basado en Competencias (HTKM), incluye los principales términos que permiten caracterizar las competencias organizacionales y su relación directa a posiciones y roles en la organización; y (5) El modelo de TIC's (TKM) que permite representar aspectos relacionados con la infraestructura tecnológica de las organizaciones, incluyendo hardware, software, ambientes de soporte, metodologías requeridas para el despliegue de tecnología de soporte a la gestión del conocimiento. 


\section{Modelos de análisis}

Los modelos de análisis permiten definir conocimiento esencial para orientar procesos de diagnóstico y definición del estado actual de la organización. Tres modelos de análisis han sido definidos: (1) el modelo para la Construcción de Mapas de Conocimiento (KMAPM), que toma cada uno de los aspectos que describen una organización, desde sus unidades organizacionales, sus procesos, su planeación estratégica, sus recursos humanos y los mapea agregando características de análisis desde la perspectiva de conocimiento. (2) el Modelo de Madurez en gestión del conocimiento (KMMM) provee una serie de elementos que permiten evaluar el nivel de desarrollo de la organización en la adopción de estrategias, procesos, métodos y tecnologías para la gestión de conocimiento, y la disposición que tienen las personas para integrar dentro de sus actividades la perspectiva de la gestión del conocimiento. (3) El modelo de métricas e indicadores de gestión del conocimiento (KMM\&I), que provee una serie de elementos que permiten evaluar el progreso en el logro de objetivos de la gestión del conocimiento y su impacto sobre los indicadores de gestión de la organización. Las métricas soportan la toma de decisiones y la comunicación a través de la organización para entender el progreso, eficiencia, valor y alineación estratégica de iniciativas de gestión del conocimiento [29].

\section{Modelos de gestión de experiencias (EMM)}

Los modelos de gestión de experiencias permiten la gestión de experiencias en la forma de casos particulares de la ejecución de procesos del negocio, ejecución de estrategias, cambios organizacionales, problemas y soluciones, los métodos de resolución de problemas y las lecciones aprendidas. Dos modelos han sido definidos en la librería de modelos: (1) el modelo de experiencias basado en casos (CASEKM), que permite representar conocimiento adquirido en la ejecución particular de un proceso y sus actividades, considerando los problemas y soluciones de la ocurrencia, las lecciones aprendidas, los métodos de resolución de problemas, el conocimiento del dominio involucrado, y el conocimiento de inferencia que soporta la toma de decisiones de un caso particular; y (2) el modelo de conocimiento del cambio (ChangeKM), que permite representar experiencia en la forma de cambios en la forma de hacer las cosas de la organización, y el conjunto de hechos o eventos que motivan dicho cambio.
Modelos de conocimiento para unidades organizacionales específicas

Corresponden a modelos que permiten estructurar conocimiento sobre aspectos específicos para soportar la operación de determinadas unidades organizacionales. Tres modelos están definidos en la librería de modelos: (1) el modelo de proyectos (PKM), que estructura conocimiento sobre proyectos de la organización; (2) el modelo de clientes (CKM), que permite estructurar conocimiento sobre clientes de la organización; y (3) el Modelo de Productos y Servicios (P\&SKM), que permite representar conocimiento sobre los productos y servicios de la organización objetivo.

\section{Modelos de valoración del conocimiento (KVM)}

Los modelos para la valoración del conocimiento (KVM) permiten la definición de medidas de valor para los activos de conocimiento de la organización y definen un marco conceptual para su inclusión en los reportes financieros. Este marco conceptual tiene en cuenta los aspectos consignados en estándares internacionales, las regulaciones contables de los países de origen de las organizaciones valoradas y propuestas propias centradas en la generación de modelos para la estructuración de conocimiento que permitan hacerlo explicito, fácilmente identificable, empaquetable y vendible.

\section{Modelos del dominio}

Los modelos del dominio se centran en los objetos/ítems del conocimiento que la organización consume/produce en la ejecución de sus procesos del negocio. Estos modelos del dominio son potencialmente distintos de una organización a otra dependiendo de su negocio. Por ejemplo, los modelos del dominio para una organización cuyo negocio es el turismo serán distintos que para una organización cuyo negocio sean los componentes electrónicos. De igual forma, distintas áreas del negocio de la organización requieren conocimiento específico de sus campos de acción.

\section{Modelos de Soporte (KMSM)}

Los modelos de soporte definen estructuras de conocimiento comúnmente empleadas en sistemas de gestión del conocimiento y que soportan estrategias de gestión del conocimiento. Los modelos de soporte incluyen el modelo de lecciones aprendidas (LLKM), el modelo de mejores prácticas (BestPracticesKM), el modelo de documentos (DocKM), y el modelo de ideas (IdeasKM). 


\section{BASE DE CONOCIMIENTO DE EXPERIENCIAS EN KARAGABI KMMODEL}

La base de conocimiento de experiencias provee métodos y estructuras que facilitan la construcción colaborativa de conocimiento en la forma de casos de ejecución de la metodología de intervención (Base de Conocimiento de Experiencias Basada en Casos), o en la forma de librerías y paquetes de conocimiento que agrupan un conjunto de instancias estándares avaladas por una comunidad o comunidades de expertos (Librerías de Conocimiento Estándar). $\mathrm{Al}$ acumular conocimiento se busca reducir tiempos y costos en el diseño y despliegue de organizaciones orientadas a conocimiento, facilitando la localización y reutilización de casos similares, evaluados, valorados y contextualizados, así como de conocimiento estándar ampliamente aceptado por comunidades de expertos.

\section{Base de conocimiento de experiencias basada en casos.} La base de conocimiento de experiencias basada en casos está basada en los modelos para la gestión de experiencias de la librería de modelos (EMM) y permite la especificación de casos de ejecución de los procesos, subprocesos, y actividades de la metodología de intervención, asociando a cada caso las lecciones aprendidas, ideas, problemas y los métodos de resolución de problemas. La base de conocimiento de experiencias actual corresponde a cuatro casos prácticos de ejecución del modelo y una serie de casos teóricos basados en la evaluación de la respuesta del modelo a casos ya desarrollados. Algunos de estos casos corresponden a: (1) La Construcción de un Mapa de Conocimiento para Cadenas Productivas conformadas como Redes Sociales de Conocimiento; (2) el Diseño e Implantación de un Modelo de Gestión del Conocimiento para la Red de Parques Tecnológicos del Software de Colombia; (3) el Diseño e Implantación de un Modelo de Gestión del Conocimiento para la Corporación Incubadora de Empresas de Software de Popayán, ParqueSoft Popayán, y (4) el Diseño e Implantación de un Modelo de Gestión de Conocimiento para una entidad del sector productivo en el departamento del Cauca, Colombia. Una descripción más detallada de algunos de los casos es presentada más adelante en la sección "experiencia en su aplicación".

Librerías de conocimiento estándar. Las librerías de conocimiento permiten almacenar conocimiento estándar avalado por una comunidad o comunidades de expertos. Karagabi KMModel cuenta con una serie de librerías de conocimiento estándar, que han sido creadas como resultado de conocimiento de casos particulares de aplicación de la metodología de intervención o por recomendación directa de expertos. El equipo responsable de la introducción de iniciativas de gestión del conocimiento en la organización puede integrar en sus diseños organizacionales este conocimiento estándar, o acceder a casos de ejecución de la metodología de intervención para detectar posibles variaciones o nuevos elementos que hayan sido definidos en condiciones particulares. Los expertos y consultores que usan el modelo pueden integrar sus propias librerías para definir conocimiento aceptado en comunidades limitadas, como por ejemplo en sus propias organizaciones. Las librerías actuales del modelo Karagabi KMModel corresponden a:

Librería genérica de organizaciones basadas en conocimiento. Esta librería cuenta actualmente con elementos de librería que definen: (1) roles organizacionales orientados a gestión del conocimiento incluidos el gestor del conocimiento, administrador de conocimiento, analista de conocimiento, ingeniero de conocimiento, gerente de proyectos de conocimiento; (2) unidades organizacionales responsables de la gestión del conocimiento incluidos el departamento de gestión del conocimiento y el departamento de gestión del recurso humano; (3) procesos del negocio de gestión del conocimiento, incluyendo los propios procesos definidos por la metodología de intervención, procesos para la gestión estratégica de conocimiento, la gestión de proyectos de conocimiento, y procesos operativos de soporte a la creación, adquisición, organización, almacenamiento, distribución y aplicación de conocimiento; (4) infraestructura tecnológica de soporte a estrategias basadas en comunidades de práctica, lecciones aprendidas, gestión de experiencias; (5) otros elementos que direccionan aspectos relevantes sobre estrategias de la gestión del conocimiento.

Librería de organizaciones en red. Esta librería cuenta actualmente con elementos de librería que definen: (1) roles organizacionales incluidos las instituciones generadoras de políticas, organizaciones interfaz, organizaciones de investigación y desarrollo, organizaciones empresariales; (2) roles de gestión del conocimiento, incluidos el gestor del conocimiento y el gerente de proyectos de gestión del conocimiento, (3) procesos de gestión del conocimiento incluidos el proceso formal e informal de Transferencia de conocimiento, Combinación y Generación de conocimiento; (4) Procesos del negocio convencionales de organizaciones en red como los procesos de articulación con el entorno, procesos de comunicación corporativa, asociatividad y fortalecimiento empresarial, inteligencia competitiva y gestión tecnológica. 


\section{EXPERIENCIA EN SU APLICACIÓN}

Durante el proceso de definición y consolidación de Karagabi KMModel han sido desarrollados proyectos que han permitido diseñar, aplicar y validar distintos componentes del modelo, así como alimentar la base de conocimiento de experiencias. A continuación se describirán dos de los casos más representativos:

Construcción de un mapa de conocimiento como aporte a la consolidación del sistema regional de innovación del Cauca

Este caso corresponde a la aplicación del modelo Karagabi KMModel para la definición de un modelo organizacional orientado a conocimiento de algunas cadenas productivas pertenecientes a estrategias identificadas dentro del Sistema Regional de Innovación del departamento del Cauca, Colombia. Los siguientes son los resultados por fase:

Auditoría de conocimiento. Fueron desplegados los procesos para la construcción del mapa de conocimiento, y el análisis de las estrategias, estructuras, procesos y la cultura de la gestión del conocimiento de las cadenas productivas. La construcción del mapa de conocimiento permitió caracterizar los principales procesos productivos: producción, transformación, comercialización y consumo; actores: directos e indirectos, y activos de conocimiento ofertados y demandados, así como los flujos de conocimiento e información relacionados especialmente con la provisión de diferentes servicios de apoyo: Insumos, Asistencia Técnica, Investigación y Apoyo Financiero. El análisis de la cultura de conocimiento permitió identificar la desarticulación entre actores de la cadena productiva por desconocimiento de ofertas y demandas de conocimiento, y una filosofía de funcionamiento de cadena convencional, con competencia entre los mismos productores, y la ausencia de una orientación hacia el logro de objetivos comunes. La etapa de auditoría de conocimiento evidenció algunas características de las cadenas productivas que las diferencian de una organización convencional. Las cadenas productivas son complejas, con actores heterogéneos, autónomos, interdependientes, con intereses encontrados y sin mando unificado.

Diseño de la organización orientada a conocimiento. Las características asociadas a las cadenas productivas identificadas en la fase de auditoría son análogas con las que presenta una red social, en virtud de lo cual las cadenas productivas se analizaron como redes sociales de conocimiento, definiendo los procesos y estructuras para la gestión de conocimiento requeridas para promover la generación de aprendizaje social e innovación, y contribuir así con su conformación como cadenas de valor y a su vez con la consolidación del Sistema Regional de Innovación. Se diseñaron los procesos de generación, transferencia, combinación y apropiación de conocimiento, y los procesos de comunicación entre los actores de las cadenas. El proceso incluyó también la definición y caracterización de procesos fundamentales para la generación de valor, como lo son la inteligencia competitiva, la articulación con el entorno, la asociatividad y fortalecimiento empresarial, y la gestión tecnológica. Se reconocieron cuatro tipos principales de actores: las organizaciones generadores de políticas, las organizaciones interfaz, los actores empresariales, y las organizaciones de I+D. Cada uno de estos actores asume diferentes roles en los procesos de gestión de conocimiento modelados.

Definición y despliegue de la infraestructura tecnológica. Para apoyar el despliegue de las estrategias y procesos diseñados se determinó la construcción de un portal de conocimiento al cual se integraron los modelos núcleo, así como el modelo de mapa de conocimiento de Karagabi KMModel, con el propósito de hacer accesible a los distintos actores relacionados con las cadenas productivas el conocimiento relevante, habilitando su generación, adquisición, distribución, e intercambio.

Diseño e implantación de un modelo de gestión del conocimiento para ParqueSoft Popayán. En este caso se aplica Karagabi KMModel para soportar el diseño y despliegue de un modelo de gestión del conocimiento enfocado en las relaciones interinstitucionales de ParqueSoft Popayán.

Auditoría de Conocimiento. El proceso para la construcción del mapa de conocimiento permitió la identificación de una serie de activos de conocimiento de la organización como su red de contactos (clientes y proveedores), la experiencia en la formulación y gestión de proyectos, en la aplicación y despliegue de modelos de gestión tecnológica en incubadoras de empresas de base tecnológica, la experiencia y conocimiento en la dirección estratégica de incubadoras y su red de aliados, entre otros. Un análisis detallado del estado actual de la gestión del conocimiento y de la cultura organizacional identificó que no se disponía de estructuras, procesos y roles formales de gestión del conocimiento, y que la no existencia de una cultura orientada a conocimiento y de una visión compartida de los beneficios del trabajo conjunto con su red de aliados eran factores importantes que podrían comprometer el despliegue de las iniciativas futuras. 
Diseño de la Organización del Conocimiento. Para adelantar esta fase se analiza la organización como parte de una red conformada por sus clientes, proveedores y aliados y se accede a la base de conocimiento de experiencias para determinar los procesos, estructuras y roles que podrían ser integrados al modelo de gestión del conocimiento de ParqueSoft Popayán. Se definen dos procesos esenciales, el proceso de transferencia formal e informal de conocimiento, y el proceso de combinación de conocimiento entre actores de la red. Son definidos tres roles fundamentales para desplegar los procesos de gestión del conocimiento, el rol de gestor del conocimiento, el de administrador de proyectos de conocimiento y el de administrador de sistemas de conocimiento. Se desestima la posibilidad de crear un departamento independiente y se relacionan los roles a la estrategia de talento humano. Adicionalmente se integran a los procesos de comunicación corporativa y de articulación con entidades de apoyo, actividades y roles de gestión del conocimiento.

Definición y despliegue de Infraestructura Tecnológica. De la librería de sistemas y aplicaciones y como resultado de la ejecución de esta fase se identificaron las siguientes soluciones como potenciales a ser desplegadas. La selección incluye además aspectos como costos, disponibilidad y nivel de desarrollo. (1) Intranet y Extranet Corporativa, (2) Sistema de Gestión de Comunidades Virtuales para el soporte al intercambio informal de conocimiento, (3) Plataforma de E-Learning para la articulación con la academia.

\section{CONCLUSIONES}

Karagabi KMModel ha sido desarrollado en el marco de proyectos de investigación y desarrollo que buscan la construcción de organizaciones orientadas a conocimiento. Cada proyecto ha permitido la construcción, validación y evolución de componentes específicos del modelo propuesto. Su aplicación en organizaciones individuales, así como redes de organizaciones, con distintos niveles de madurez, y problemas culturales para enfrentar la gestión del conocimiento, ha impuesto una serie de retos para los equipos de consultoría involucrados y ha permitido generar una serie de conclusiones y perspectivas de trabajo futuro. A continuación se presentan las principales conclusiones:

Karagabi KMModel es una propuesta que facilita la incorporación de modelos de gestión de conocimiento en la dinámica de las organizaciones. Su aplicación ha permitido que las organizaciones estudiadas se reestructuren, ayudándoles a definir de manera adecuada, sus procesos, estructura, motivación, competencias, y tecnología requerida para valorar, aprovechar y desarrollar sus activos de conocimiento e integrarlos en la operación de la organización.

Uno de los componentes fundamentales en Karagabi KMModel es su librería de modelos de conocimiento. El lenguaje común y compartido que proveen para representar conocimiento organizacional es aprovechado para formalizar tanto la metodología de intervención, como los resultados de cada una de las fases de su aplicación. La librería ha sido construida de acuerdo a las teorías y prácticas de la ingeniería del conocimiento y la ingeniería de ontologías, lo que ha permitido su evolución y adaptación a los distintos casos de aplicación. La posibilidad de integrar nuevos modelos, extender los existentes o especializarlos hacen de Karagabi KMModel un modelo altamente adaptable y escalable.

Los modelos de análisis son fundamentales para Karagabi KMModel. Permiten reconocer la heterogeneidad de las organizaciones, la forma en que su estructura, motivación, cultura afectan el aprovechamiento y desarrollo del conocimiento, y dan el punto de partida para el diseño de estrategias de gestión de conocimiento acordes con los objetivos estratégicos y los problemas detectados. La definición de métricas e indicadores de gestión del conocimiento, y su adición a la base de conocimiento de experiencias es un aspecto a mejorar dado que aunque se tiene un modelo para caracterizarlos, las experiencias prácticas aún no permiten llegar a un consenso sobre los más adecuados.

Karagabi KMModel provee un marco de trabajo integral para el diseño de organizaciones orientadas a conocimiento. Sus componentes acogen diferentes propuestas de la ingeniería del conocimiento, la gestión de conocimiento y la gestión organizacional, pero no están atados a ninguna en particular. Los equipos de gestión de conocimiento pueden aplicar todo el modelo como referente o pueden integrar nuevas propuestas, agregando o rediseñando procesos y actividades de la metodología de intervención, modelos de conocimiento a la librería de modelos, y alimentando la base de conocimiento de experiencias. Es una tendencia en todos los casos de aplicación que los participantes, de acuerdo a su experiencia, planteen estrategias y procesos alternativos, para orientar el proceso de diseño de las organizaciones; por ejemplo, la identificación de la necesidad de desplegar estrategias orientadas a la gestión del talento humano por competencias en algunos de los casos de estudio permitió la definición de un conjunto de procesos, modelos de conocimiento y mejores prácticas para abordar esta perspectiva, 
los cuales fueron formalizados empleando los conceptos y notaciones de los modelos núcleo, e integrados como parte de Karagabi KMModel.

La acumulación de experiencias puede generar oportunidades importantes en el desarrollo futuro del modelo al permitir incorporar métodos, metodologías y conocimiento avalado por expertos como resultado de cada caso de estudio. La experiencia almacenada permitirá hacer cada vez más robusto el modelo reduciendo tiempos y costos de su aplicación. La integración de infraestructura software para la identificación de casos similares y su adecuación a nuevos casos de aplicación representa un reto futuro como herramienta de apoyo a los equipos de consultoría.

Es fundamental integrar al modelo las herramientas informáticas para la captura de conocimiento basado en los modelos de conocimiento construidos. Herramientas informáticas para apoyar la construcción de mapas de conocimiento, la caracterización de procesos, estrategias y estructuras, la gestión de indicadores entre otras, son esenciales para facilitar en proceso de diseño de organizaciones orientadas a conocimiento.

\section{AGRADECIMIENTOS}

Karagabi KMModel ha sido desarrollado por la empresa TOTEMS LTDA ${ }^{8}$ [30], con el apoyo del grupo de investigación en ingeniería del software, IDIS, de la Universidad del $\mathrm{Cauca}^{9}$, Colombia; el Instituto Colombiano para el Desarrollo de la Ciencia y la Tecnología "Francisco José de Caldas", COLCIENCIAS ${ }^{10}$; el Ministerio de Comercio, Industria y Turismo, a través del Fondo Colombiano de Modernización y Desarrollo Tecnológico de las Micro, Pequeñas y Medianas Empresas, FOMIPYME ${ }^{11}$; y el Servicio Nacional de Aprendizaje, SENA $^{12}$. Los proyectos que han contribuido al desarrollo y evolución de Karagabi KMModel son: (1) Entorno Colaborativo de Apoyo a la mejora de procesos para la Industria del Software Colombiana (Código 3531-40320708, financiado por Colciencias); (2) "Infraestructura software para la gestión, distribución, uso y aplicación de conocimiento organizacional" (Código 41281418008, financiado por COLCIENCIAS); (3) "Desarrollo de un Mapa de Conocimiento como aporte a la Consolidación del Sistema Regional de Innovación del Cauca" (Código 11240217444, financiado por COLCIENCIAS);

\footnotetext{
8 http://www.totems-software.com

9 http://www.unicauca.edu.co/idis/

$10 \mathrm{http}: / / \mathrm{www}$. colciencias.gov.co

11 http://www.mincomercio.gov.co

12 http://www.sena.edu.co
}

(4) "Estrategia Colectiva de Gestión Tecnológica Para la Red de Parques Tecnológicos del Software" (Financiado por COLCIENCIAS); (5) "Construcción colaborativa de ParqueSoft Popayán como una organización inteligente, haciendo uso de herramientas y procesos de trabajo colaborativo y de gestión del conocimiento" (Financiado por el SENA); (6) Proyectos de fortalecimiento empresarial para la empresa TOTEMS LTDA, financiados por el SENA y FOMIPYME.

\section{REFERENCIAS}

[1] I. Nonaka and H. Takeuchi. "The knowledge creating company: How Japanese companies create the dynamics of innovation". Oxford University Press. London. 1995.

[2] T.H. Davenport and L. Prusak. "Working Knowledge: How organizations manage what they know". Harvard Business School Press. Boston. 1997.

[3] R. Andreu y S. Sieber. "La gestión integral del conocimiento y el aprendizaje". Economía Industrial. No 326, pp. 63-72. 1999.

[4] M. Uschold, M. King, S. Moralee and Y. Zorgios. "The Enterprise Ontology". The Knowledge Engineering Review. Vol. $13 \mathrm{~N}^{\circ}$ 1, pp. 31-89. 1998.

[5] S. Decker, M. Daniel, M. Erdmann and R. Studer. "An enterprise reference scheme for integrating Model based knowledge engineering and enterprise modeling". 10th European Workshop on Knowledge Acquisition, Modeling and Management, EKAW'97. Lecture Notes in Computer Science. Vol. 1319. 1997.

[6] G. Papavassiliou and G. Mentzas. "Knowledge modelling in weakly structured business processes". Journal of Knowledge Management. Vol. $7 \mathrm{~N}^{\circ} 2$, pp. 18-33. 2003.

[7] G. Schreiber, H. Akkermans, A. Anjewierden, R. de Hoog, N. Shadbolt, W. Van de Velde and B. Wielinga. "Knowledge Engineering and Management, the CommonKADS Methodology". The MIT Press. Londres, Inglaterra, p. 471. 1999.

[8] A. Gómez Pérez, M. Fernández and O. Corcho. "Ontological Engineering". Springer Verlag. London. 2003. 
[9] W.M.P. van der Aalst, A.H.M. ter Hofstede and M. Weske. "Business Process Management: A Survey". International Conference on Business Process Management (BPM 2003). Lecture Notes in Computer Science. Vol. 2678, pp. 1-12. 2003.

[10] M. Smits and A. de Moor. "Effective knowledge management in knowledge - intensive organizations". Proceedings of Fifth European Conference on Organizational Knowledge, Learning, and Capabilities. Innsbruck, Austria. 2004.

[11] S. Staab, R. Studer, H. Schnurr and Y. Sure. "Knowledge Processes and Ontologies". IEEE Intelligent Systems. Vol. $16 \mathrm{~N}^{\mathrm{o}}$ 1, pp. 26-34. January, 2001.

[12] G. Papavassiliou, S. Ntioudis, G. Mentzas and A. Abecker. "Business Process Knowledge Modelling: Method and Tool'. Proceedings of $13^{\text {th }}$ International Workshop on Database and Expert Systems Applications, pp. 138-142. September 2002.

[13] G. Mentzas, D. Apostolou, R. Young and A. Abecker. "Consolidating the Product Versus Process Approaches in Knowledge Management: The Know-Net Approach”. PAKeM2000. Manchester, United Kingdom. 2000.

[14] T. Kucza. "Knowledge Management Process Model”. VTT Publications 455. Technical Research Centre of Finland. Espoo, Finland, p. 111. 2001.

[15] L. Edvinsson. "Developing intellectual capital at Skandia”. Long Range Planning, Vol. $30 \mathrm{~N}^{\mathrm{o}} 3$, pp. 366-373. June 1997.

[16] V. Kochikar. "The Knowledge management maturity model - a staged framework for leveraging knowledge". Proceedings of KM World 2000. September 2000. Fecha de consulta: Febrero 2006. URLs: www.infy.com/knowledge_capital/ knowledge/KMWorld00_B304.pdf

[17] O. Paulzen and P. Perc. "A maturity model for quality improvement in knowledge management". Proceedings of Enabling Organizations and Society through Information Systems (ACIS-2002), pp. 243253. 2002.

[18] R. Shevlin, R. Maney, J. Sawyer and B. Edwards. "The stages of knowledge management". The
Forrester Report - Leadership Strategies. Forrester Group. Vol. 3 No 2. 1997.

[19] H.T. Larsen, J. Mouritsen and P.N. Bukh. "Intellectual capital statements and knowledge management: Measuring, reporting and acting". Australian Accounting Review. Vol. $9 \mathrm{~N}^{\mathrm{o}} 3$, pp. 15-26. 1999.

[20] L. Edvinsson. "Developing Intellectual Capital at Skandia". Long Range Planning. Vol. 30 N$^{\circ} 3$, pp. 266-373. 1997.

[21] Fundación Chile. Competencias Laborales para Chile 1999-2004. Fecha de consulta: Febrero 2006. URLs: www.chilecalifica.cl/prc/n-1971comp_lab_1.pdf

[22] Corpoeducación. Competencias Laborales: Base para mejorar la empleabilidad de las personas. Bogotá, D.C. Agosto 2003. Fecha de consulta: Febrero 2006. URLs: www.oei.es/etp/competencias_ laborales_base_mejora_empleabilidad_personas. pdf

[23] G. Iazzolino and R. Pietrantonio. "Auditing the organizacional knowledge through a Balanced Scorecard-based Approach". International Conference on Knowledge Management in Asia Pacific (KMAP 2005). November 2005.

[24] T.W. Lauer and M. Tanniru. "Knowledge Management Audit - A Methodology and Case Study". Australasian Journal of Information Systems. Vol. $9 \mathrm{~N}^{\mathrm{o}}$ 1, pp. 23- 41. 2001.

[25] D. Grey. "Knowledge Mapping: a Practical Overview". SWS Journal. 1999. http://smithweaversmith.com/ knowledg2.htm

[26] T. Davenport and L. Prusak. "Working Knowledge - How Organizations Manage what they Know". Harvard Business School Press. USA, p. 224. 1998.

[27] R. Bergmann. "Experience Management: Foundations, Development Methodology, and Internet-Based Applications". Springer-Verlag New York, Inc. New Jersey, USA. 2002.

[28] Object Management Group. "Business Motivation Model Specification”. Septiembre 2007. Fecha de consulta: Octubre 2007. URLs: www.omg.org/ docs/dtc/07-08-03.pdf 
[29] Department of Navy (USA). Metrics Guide for Knowledge Management Initiatives. 2001. Fecha de consulta: Agosto 2006. URLs: www.susanhanley. $\mathrm{com} /$ sitebuildercontent/sitebuilderfiles/metricsguide. pdf
[30] A. González and C. Joaquí. "Karagabi KMModel V1.0". TOTEMS LTDA. Popayán, Colombia. 2007. 